\title{
Atlas - Examining the Wider Context of Assistive Robotics
}

\author{
H. D. Doran ${ }^{1}$. S. Neumann². H.K. Becker². A. Kollmar². S. Misoch ${ }^{3}$. C. Pauli ${ }^{3}$ S. Müller ${ }^{4}$, F. Hannich . \\ ${ }^{1}$ Institute of Embedded Systems, Zürich University of Applied Sciences, Winterthur, Switzerland. \\ ${ }^{2}$ Institute of Occupational Therapy, Zurich University of Applied Sciences, Winterthur, Switzerland. \\ ${ }^{3}$ Interdisciplinary Competence Centre for Ageing IKOA-FHS, University of Applied Sciences St. Gallen, St. Gallen, Switzerland \\ ${ }^{4}$ Institute of Marketing Management, Zurich University of Applied Sciences, Winterthur, Switzerland \\ \{donn, neus, behe,kola\}@zhaw.ch, \{cora.pauli, sabina.misoch\}@.fhsg.ch \{mues, hanf\}@.zhaw.ch
}

Keywords: $\quad$ Elderly care robotics, Assisted living, Assistive robotics

\begin{abstract}
We examine the proposition of a stationary assistive robot arm in the kitchen. Based on a preliminary business plan and with the aim of generating engineering requirements, a multi-disciplinary project was established to examine the wider ramifications of such assistive technology in the household, in a Swiss context, in the fields of health and social wellbeing. Additionally the engineering aspects as well as the business aspects were examined. We detail both the individual methodologies used in this study, the results achieved and discuss the results in a wider context.
\end{abstract}

\section{INTRODUCTION}

\subsection{Motivation}

Over the course of a masters level business plan creation week a group of engineering students produced a well-argued business plan for a kitchen assistant robot they called ATLAS. Given the current interest in assistive robotics but cognisant of the fact that complete understanding of the application area was out of scope for the normal robotics engineer it was decided to construct a multi-disciplinary consortium to examine the proposal in a more structured manner. This paper describes the results achieved (Neumann 2016).

The novelty in this paper is that we focus on the elderly still resident in their own homes in the context of enabling them to remain there longer rather than servicing those in care-homes or those with constrained movement abilities.

\subsection{Consortium and Task}

The driving task was to establish engineering requirements for a robotic assistive arm in the kitchen guided by the scenario of an elderly person cooking. The task of the consortium was to establish whether and in what form a robotic assistive arm was an acceptable accessory for elderly people in their household kitchens. The consortium was designed to complement engineering in domains where engineering researchers traditionally have no specific knowledge in this case business, health and social research. In addition to the research consortium, expertise from the Swiss national organisation representing interests of the elderly, Pro Senectute, was requested and granted. Funding was granted by the Walder Stiftung.

\subsection{Value of Paper and Structure}

A large number of assistive robotic projects are generated by engineering research and less by market requirements, an exception to this are assistive robotics for special needs patients. To a large part, the value of this paper is an indication of how projects with collaborators from different disciplines can help generate engineering specifications or, as in our case, show that an imagined product might not find a market. We also show that user requirements are a qualitative and not quantitative research area and different approaches, whilst converging on a common solution, offer greater understanding of the problem domain as well as otherwise easily missed nuances so this paper also serves to illustrate the techniques used by other disciplines to interested researchers.

This position informs the general structure of the paper. The next section deals with the engineering element of this project, Section III, which is further sub-divided by competence domain, examines the problem space by that domain. The final section draws appropriate conclusions and details future work suggestions.

\subsection{Initial Engineering Position}

The engineering work consisted of three main tasks, the establishment of the proposed system 
capabilities, an advisory position within the consortium and the development of a mock-up system.

\subsubsection{System proposal}

In order to give the other researchers a visualisation of the system preliminary usability studies were commissioned. The results, which were presented to the other researchers, are shown in the figures below. The initial proposition was that there would be a unit in the kitchen dedicated to the heavy elements used in cooking, plates, pounds of sugar or rice etc. and the oven itself, or more specifically the contents thereof (Figure 1).

The morphology of the assistive arm was also considered important. The initial assessment considered factors such as number of joints and structure (Figure 2), the rendering of the arm and gripper, the form and technology of the gripper (Figure 3) and whether the rendering would be part of the control system of the arm or not. Significant engineering uncertainties were associated with the rendering of the arm and the gripper, various ideas from artificial skin to wood were considered before it was decided to hand this issue over to researchers from another domain.

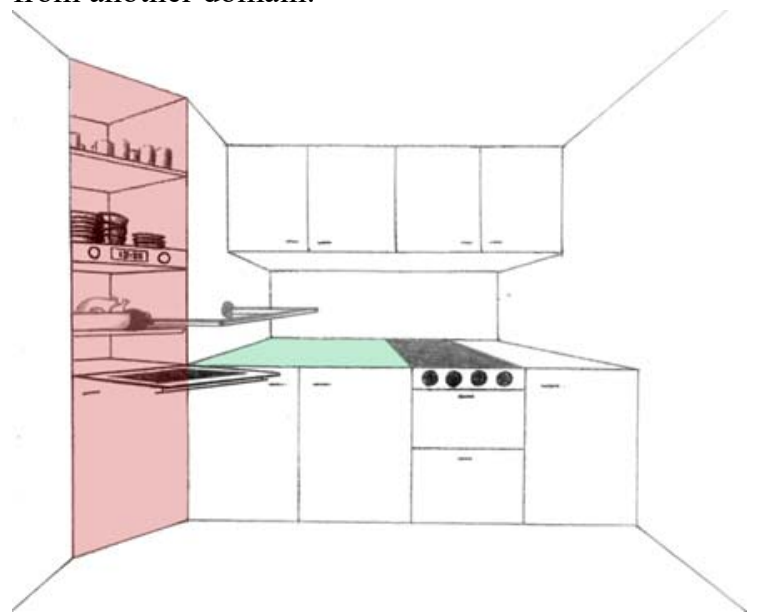

Figure 1: Kitchen setup with assistive robot arm attached to wall. The vertical shaded area is a kitchen unit designed for access by the assistive arm. The arm is shown reaching for a roast chicken in the oven. The shaded horizontal area is the workspace accessible by the assistive arm.

\section{INTERDISCIPLINARY RESEARCH}

\subsection{Further Engineering Work}

Independently of the main body of work an

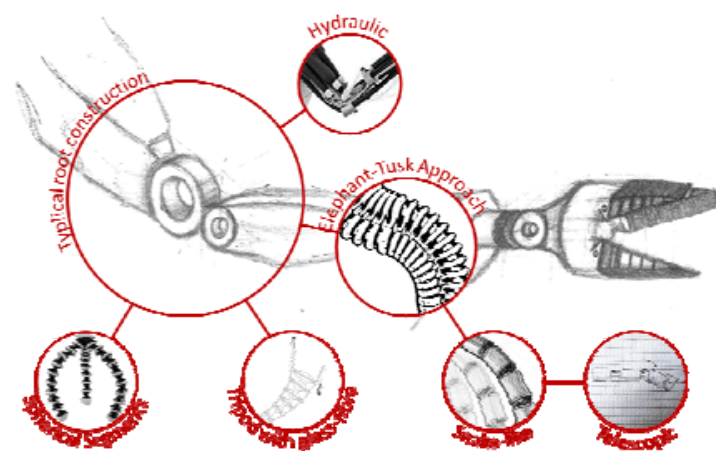

Figure 2: Possible arm morphologies.

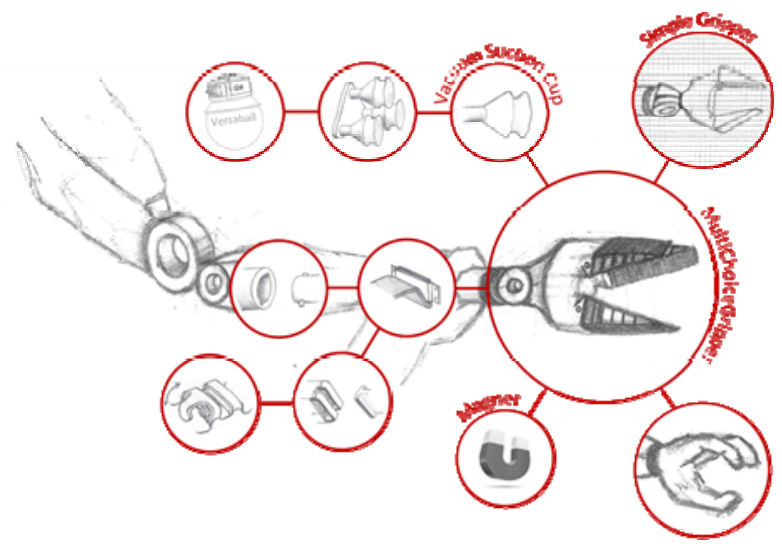

Figure 3: Possible gripper morphologies.

engineering mock-up of a possible assistive arm was commissioned (Bucher 2016). The work was temporally staggered with that of the other research groups. No results of this body of work were shared with the non-engineering researchers for several reasons. The first was because of uncertainties of the design work. Engineering was cognisant of the fact that because the initial design was starting from the same knowledge base as the rest of the project, several design iterations would be necessary to reach acceptable price and optic points.

It was also feared that early insight to the engineering ideas would create a prejudice on the part of the other researchers who may have less realistic expectations of the ability of engineering to create an optically acceptable prototype. Another reason was to avoid anticipating a solution with the other researchers. A fourth was to avoid any specific discussions in focus groups about the optics of the device. This mock-up was advanced as far as a simulation and the drawings and specifications for production of a prototype including pricing (Figure 4.) 
The general features of the assistive arm are a twoaxis arm with a spindle drive for the vertical direction. The arm can carry $10 \mathrm{~kg}$ load and deposit trays on the work surface on the left of the sink. A simple hook-like gripper was chosen (Figure 5). The unit mechanics were designed to allow a self-stow along the top of the adjoining kitchen elements.

Initial costing for the mechanics, without the cladding to suit the kitchen design, without the shelf/oven elements, and without the electronic control units but including the drives, was estimated to be around 4'100 CHF calculated with one-off pricing. Based on these criteria the finished design, again without cladding and shelf and oven elements, could retail for about $15^{\prime} 000 \mathrm{CHF}$.

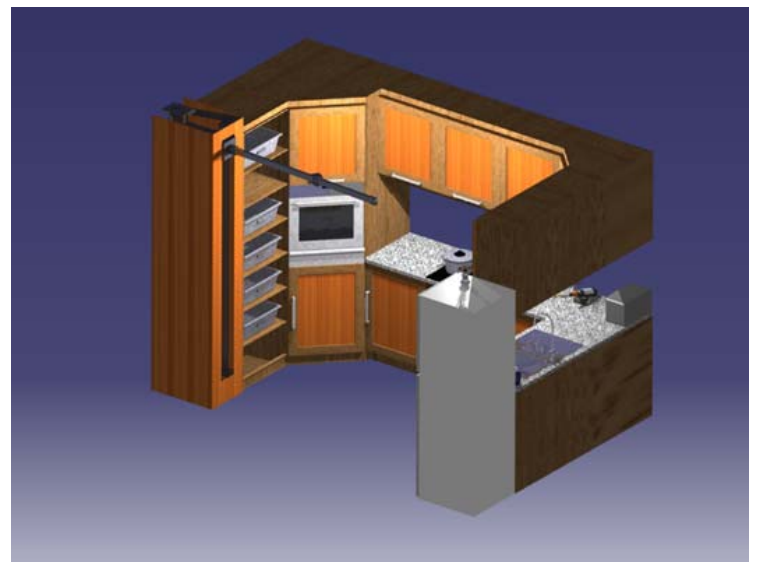

Figure 4: Simulation of an assistive kitchen arm (far left). The arm moves up and down and can place/extract trays from the shelving beside it onto the work surface as well as move objects in and out of the oven.

\subsection{Social Significance of the Kitchen}

\subsubsection{Motivation}

The introduction of a significant technical investment into the living space may cause a change as the family learn to live with, and around, such a device. Electric light or the television are most obvious examples but the introduction of an assistive robot arm in the kitchen may, or may not, also represent a dislocation in the accepted fabric of rooms and their use by a family and this was the question being asked in this part of the study. This is a fundamentally different question as to the acceptance of such a unit as a technical object as asked in the next part of the study where the question was based in reference to the failing physical abilities of the elderly and the microconditions under which such a unit might be used.

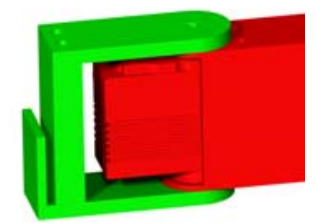

Figure 5: Chosen gripper for the mock-up. There is a drive integrated in the joint and the end is designed to hook into the trays and oven door.

\subsubsection{Methodology}

Two group interviews were organised to establish the significance of the kitchen in a social context for the family. The group interview is a structured interview process which consists of the answering of pre-prepared open or partially standardised questions by the interviewees. There is the possibility for the interviewees to react to and discuss the answers of the other participants (Misoch 2015).

The first group consisted of two women and one man between the ages of 70 and 80 all of whom were married and all of whom have children and grandchildren. In both cases, the women were responsible, as homemakers, for kitchen and household.

The second group consisted of five women and two men between the ages of 75 and 89 . These participants all had reduced motor-function due to arthritis, rheumatism, after-effects of strokes, failing strength in back and arms etc. and were all living in separate flats in the same housing scheme with each flat designed for people of that general health category.

\subsubsection{Scope and Results}

The three themes researched were the kitchen, cooking and problems cooking or in the kitchen.

\section{Kitchen.}

In all cases the kitchen was considered more of a functional room than a social room and like most functional rooms it was important that everything could be properly stored, and was ready to be used when required. It represented a personalised optimisation between aesthetics and functionality in that kitchen machines that were used on a daily basis might occupy permanent space on a kitchen 
counter. New technology in the kitchen was in all cases acceptable.

The idea of the purely functional room was often qualified depending on the available space where children and husband could talk about the day whilst the mother was cooking.

The study showed that the meaning of the kitchen as a social/functional space did not change as much as the act of cooking during the aging time-span and that the participants increased their reliance on kitchen machinery, for instance automated breadslicers, as their physical robustness deteriorated as they aged.

\section{Problems Working in the Kitchen}

Issues with working in the kitchen with increasing age and the effects, after effects and slow recovery from diseases could be divided into four categories. Pain whilst cooking, decreasing strength in hands, arms and back, changing body sizes and declining strength during cleaning after cooking.

Pain was often ignored especially when preparing meals for the partner whereby the scope of the individual meals and the range of foodstuffs prepared was significantly reduced, based in part on the avoidance of pain. The decline in strength, be it from increasing age or as a result of illness, resulted in the increased use of electrical and mechanical appliances and the avoidance of certain heavy objects, particular pans or pots etc. In both cases the work-flow, so to speak, was optimised and carried out at a slower pace to cope with the hindrances.

The changes in body size with age generally effected the ability to reach things in higher cupboards and resulted in a rearrangement of the contents of the storage in the kitchen, which also resulted, in one case, with the dishwasher being used to store things.

Finally cleaning after cooking was considered so problematic that this becomes a reason not to cook for guests, an effect more pronounced with individuals who place importance on cleanness and orderliness.

\section{Cooking}

Also questioned was the change of the importance of cooking as such in a household. In summary whilst women of that generation may or may not have experienced fulfilment in cooking, that function really began with the founding of a family. The importance of cooking as a social act declines to a certain extent, especially when the children leave home, so whilst regular preparation of meals, and shopping for ingredients, helps structure the day, the number of complex meals prepared at home tends to reduce to special occasions. While this also results in a decline in the ability to manage the cooking of larger (multi-course) meals it is not seen as a loss mainly due to the change in appetite and the increase in available time that reduction of cooking effort affords. Of course the option of a restaurant for special occasions was frequently mentioned.

\subsection{Acceptance Study}

\subsubsection{Motivation}

Having established that a robotic assistive arm is not necessarily an intrusion in the personal sphere of the inhabitants of the dwelling where it is to be installed, it is important that the articulation of the robot addresses the needs of the users. Given the physiological nature of the intended function, in other words the substitution of specific human movements due to weakness and pain, health researchers examined the requirements for acceptance of a robotic arm in a kitchen environment.

\subsubsection{Methodology}

In a first of two work packages, a literature study was undertaken to identify the difficulties the elderly, or disabled have while working in the kitchen and from which requirements on an assistive robot arm can be established. Then the acceptance criteria of new technology in the kitchen was examined and finally a review and critical analysis of research projects in this field compiled.

The conclusions of this work package were to be used to inform and guide the constitution and questioning of focus groups that were to be organised in the second work package.

The methodology of the first part was to use databases to find papers on kitchen robotics, unsurprisingly few papers could be found on this theme so the search was widened to include general household robots. After filtering out double hits and those (13) dealing with robot technicalities without discussing user-experience, 27 papers were included for further study. From these information and data was gleaned concerning the problems and limitations of the elderly in everyday situations, this was used to generate initial requirements for household/kitchen robotics. In the third step the acceptance criteria for technology in 
household/kitchen robotics was determined, with particular focus on apprehensions and fears/suspicions of the target group with respect to the Technology-Acceptance Model (TAM, Venkatesh 2000).

The methodology applied in the second work package was to (attempt to) form two focus groups, one consisting of elderly persons and the other of people with constrained movement abilities. Recruitment was very difficult, interestingly enough those with constrained movement abilities approached were not interested in technical solutions believing that they were so constrained that it would be better for them to learn to live without technical help. For the elderly focus group only four people could be recruited.

\subsubsection{Scope and Results}

\section{Literature Review}

The literature review resulted in the understanding that cleaning help was the most required assistance in the household (Pigini 2012, Beer 2012, Ng 2012) followed by reaching for things and carrying them (Choi 2009, Telson 2013, Beer 2012, Pigini 2012, Fischinger 2016) or holding things in an assistive capacity (Pigini 2012, Beer 2012). Since potential users, through the literature, generally accept that that a robot could be useful in a household, the activities with the planned focus groups would serve to confirm the literature and help inform the necessary acceptance criteria which can be structured according to the TAM.

The TAM informed criteria for questioning the focus groups - in this case perceived usefulness; perceived ease; image and technology experience. The TAM does not recognise differences between the sexes but Brodbent et. al. (Brodbent 2009) find substantial difference to what they attribute to the more emotional approach of women vis a vis the more clinical approach by men towards technology.

\section{Focus Group(s)}

Severe difficulties were encountered in trying to form two focus groups and eventually only one of seven members could be formed. The results of the literature could only be partially predicted. Far more value was placed by the participants on the difficulties of opening things (bottles, jars, tins) than moving things from A to B. In fact a long list of complaints about current bottle and tin-openers followed. So in terms of the category perceived usefulness a proposed kitchen assistive robot was given a task list that it should be able to perform which began with opening things, carrying things through cleaning tasks and ending with peeling vegetables.

The "image" results were less clear. Image in this context is the effect on a person's self-image of being dependent, in this case, on technology. The one participant with movement restrictions was more inclined to want to use a robot for a multitude of tasks whereas most other participants made it dependent on limited available third-party human resources - if human help was available however most would prefer a human to do it.

In terms of technology experience most participants had enough contact with technology not to be put off, under the conditions already mentioned, to consider using it.

The group also considered the practical issues including general functional characteristics, costs and general look and feel. It was clearly seen as a kitchen device and should be in the same category as a dish washer and/or cooker, it should be able to put itself away after performing its task and be stationary not mobile and it should look technical, the idea of it having human characteristics wasn't welcomed.

\subsection{Business Considerations}

\subsubsection{Motivation}

The business researchers examined the economic viability of such a product and attempted to identify the main influencers, those who, in this case, would influence a potential user to purchase, in this case, an assistive robot arm.

\subsubsection{Methodology}

A marketing methodology, under the assumption of a strategic decision to market a kitchen assistive arm, was applied. The marketing methodology consists of several discrete steps generating measureable output and culminating in the wellknown marketing mix of Price, Product, Place and Promotion (four Ps (Doyle 2006)). Apart from generating guidelines for the marketing and sales of any technical unit, or washing powder for that matter, the four Ps can be used to generate engineering specifications right down to packaging, logistics and maintenance policies. The derivation of the four Ps is via defining a market development strategy, which in turn is derived from determining the market aims. The marketing aims define the 
demographics, market segments, positioning of a product with respect to potential competitors

\subsubsection{Scope and Results}

From the point of view of an assistive robot arm for kitchen use the market segment are the elderly and disabled, the former the larger market by far. The potential market size was determined by the use of official statistics qualified by consideration of various factors. These factors are, amongst others, the aging characteristics of the target segment, in other words when and how severely health declines to a point where such an assistive technology becomes useful and the duration of residence in the own-flat before moving to a third-party run residence. In particular the age from 80 seems to be a critical period (in Switzerland) which sees a rapid increase in the requirement for specialised care (Höpflinger 2011, BfS 2015). Not only is that but the rate of degeneration once a health dislocation has occurred is also rapid. The ramification of this is that the market segment for an assistive robot is the age group from 65-80 but the window of opportunity for the installation of an assistive robot would appear to be small.

A further hindrance is, at least in the canton of Zürich, that the average 60 year old has lived in the same accommodation for 30 years typically in 3-4.5 room apartments, which tend to be relatively small in Switzerland at $100 \mathrm{~m}^{2}$. The length of time lived in the same space would tend to discourage complicated and inconvenient retro-fits, especially difficult and expensive in smaller kitchens (Figure 6).

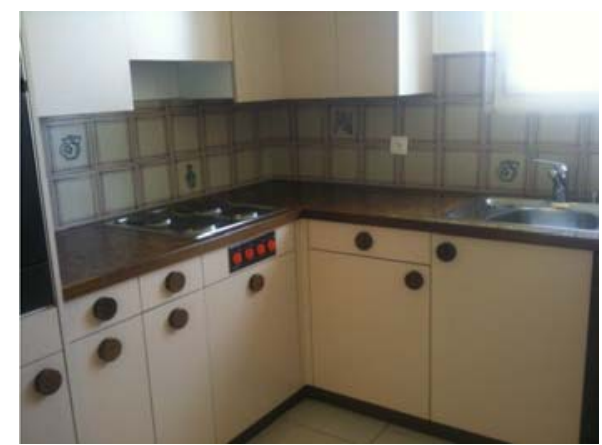

Figure 6. Typical kitchen layout in an older Swiss 3-4 room apartment (comparis.ch).

Whilst the stairs and the bathroom were considered by the target group to be the main hindrance in a currently occupied apartment, $36 \%$ of the respondents of a study categorised the kitchen as also being unsuitable for them (Höpflinger 2014).

The conclusion to be drawn here is whatever the functionality of an assistive arm, the installation aspects must be well considered.

The positioning of the solution with respect to other assistive products, both high- and low-tech and the strategic pricing options were also analysed. Unsurprisingly there appear to be no potential competitors on the market, except possibly for Moley from the UK, which intends to offer a complete programmable cooking station (Moley 2015).

Finally the sales channels and, most importantly, the potential influencers were analysed. In terms of influencers it was quite clearly determined that partners and children are the main influencers, in other words effort must be made to gain acceptance of (grown-up) children in order to sell such a device.

\section{DiscuSSION AND FUTURE WORK}

\subsection{Discussion}

There are two foci of comparison of the studies undertaken. The first is the comparison of the results of the non-engineering studies with each other and the second the combined results with the output of the initial engineering mock-up.

Whilst the three non-engineering studies complement each other they also individually offer nuances which otherwise might have gotten unnoticed and so, a more complete picture of a situation can be built. One theme is the declining importance of the kitchen as an area in the context of a family as the offspring leave the house. The kitchen tasks are reduced in their complexity and frequency. Despite this reduction in importance the kitchen is still seen as a necessary workroom by the elderly and whilst they are, prepared to invest somewhat in equipment, they exhibit health and strength declines that makes the use of such a robot merely to move stuff from $A$ to $B$ an underutilization of a potentially expensive piece of equipment.

The consequences for engineering is that a more holistic approach to a kitchen assistive robot than what was initially conceived as a pick-and-place robot is required. The assistive unit must be able to help grip things; jars, bottles, tins and the like; assist in opening them; pour the contents into a pot it has moved from storage to the stove; clean the tin for 
disposal (common in Switzerland); move pots and pans around the stove and then assist in cleaning the pots and pans when the cooking activities are finished. In this light, what is required is a sort of exo-kitchen machine rather than an assistive help for the elderly, which might even help dispel the image issue raised by the acceptance study. The three nonengineering studies clearly gave sufficient information to help understand a kitchen situation in a more structured fashion.

The three non-engineering studies also provided sufficient guidelines for engineering to design the look and feel of such a robot. A technical look-andfeel is acceptable meaning normal kitchen standards could be applied and, in our opinion, standard usability features from industrial collaborative robotics could be applied in adapted form. We also believe that the mock-up could probably be considered close to a useful first mechanical prototype.

\subsection{Future Work}

As unaware as engineering professionals may be about the methodologies employed by nonengineering researchers so are non-engineering researchers unclear about what engineers need in order to understand end-user requirements. It might be worth establishing a methodology to allow nonengineering researchers to ensure that feedback from focus groups in their research domain is as unequivocal as possible.

The research and development of an assistive robot and its use in real-world situations is not impossible but its success in the open market would, based on the presented results, be challenging. Reframing the issue scope, as suggested in the previous sub-section, along the lines of a kitchen machine might mitigate the negatives out of the presented study and accentuate the positives for surely, if such a kitchen machine becomes standard issue the elderly will benefit as well.

\section{ACKNOWLEDGMENTS}

Thanks are due to the Walder Stiftung, Zurich, for their financial support: to Pro Senectute, Zürich for their support: and to R. Pfrommer and $\mathrm{H}$. Hesselbarth, Institute of Mechanical Systems, ZHAW, for organizing and managing the engineering mock-up project. Lukas Reisinger, Institute of Embedded Systems, ZHAW, was responsible for the initial arm and gripper designs.

\section{REFERENCES}

Beer, J.M., Smarr, C. Chen, T. Prakash, A. Mitzner, T. Kemp, C. Rogers, W.A. 2012 "The Demesticated".

BfS, Bundesamt für Statistik. "Statistik der Hilfe und Pflege zu Hause - Ergebnisse 2014: Zahlen und Trends.“ 2015 Neuchâtel: BfS.

Brodbent, E., Stafford R., MacDonald B., 2009 "Acceptance of Healthcare Robots for the Older Population: Review and Future Directions," Int J Soc Robot 1:319-330.

Bucher, S. Di Fazio, L. "Kitchen-Aid", 2016. Project report ZHAW, unpublished.

Choi YS., Deyle T., Chen T., Glass J.C., Kemp CC. 2009 "A List of Household Objects for Robotic Retrieval Prioritized by People with ALS", IEEE, 11th International Conference on Rehabilitation Robotics, Kyoto International Conference Center, Japan, June 23-26.

Doyle, 2006 Marketing Management and Strategy, Prentice Hall; 4 edition (May 28, 2006)

Fischinger D., Einramhof P., Papoutsakis K., Wohlkinger W., Meyer P., Panek., Hofmann S., Köertner T., Weiss a., Argyros A., Vincze M., 2016. Robotics and Autonomous Systems 75; 60-78.

Höpflinger, F., Bayer-Oglesby, L. \& Zumbrunn, A. 2011 Pflegebedürftigkeit und Langzeitpflege im Alter. Bern: Hans Huber.

Höpflinger, F., Van Wezemael, J. (Hrsg.) 2014. Age Report III: Wohnen im höheren Lebensalter Grundlagen und Trends. Zürich: Seismo.

Misoch, S. 2015 "Qualitative Interviews.” Berlin: Walter de Gruyter.

Moley 2015 http://www.moley.com/ last accessed 16.03.2018

Neumann, S., Becker, H.K., Kollmar, A., Misoch, S., Pauli, C., Doran, H.D., Müller, S., Hannich, F. 2017. «Küchenassistenzroboter für Seniorinnen und Senioren: Bedürfnisse, Akzeptanzfaktoren und Wirtschaftlichkeit." In: G. Kempter, I. Hämmerle, (Ed). Umgebungsunterstützes Leben. Beiträge zum Usability DayXV (38-44). 15. Lengerich: Pabst.

Ng, J. Tan, O. Wong, A. Kiat, K.W. 2012. "Older Adults' Attitudes towards Homfes Service Robots," WASA, 11;26-27 2012.

Pigini, L. Facal, D. Blasi, L. Andrich, R. 2012. "Service robots in elderly care at home: Users' needs and perceptions as a basis for concepts development," Technology and Disability 24;303-311.

Telson J.,2013, Research Update- A Helping Hand in the Kitchen; PN, 38-40

Venkatesh, V. Davis, F. 2000 "A theoretical extension of the technology acceptance model: Four longitudinal field studies." Management science 46(2), 186-204. 2000 